\title{
Reduced 10-year risk of developing cardiovascular disease after participating in a lifestyle programme in primary care
}

\author{
Lena Lönnberg ${ }^{\mathrm{a}, \mathrm{b}}$ (D), Elin Ekblom-Bak ${ }^{\mathrm{c}}$ (D) and Mattias Damberg ${ }^{\mathrm{a}, \mathrm{b}}$ \\ ${ }^{a}$ Center for Clinical Research, County of Västmanland, Uppsala University, Västerås, Sweden; ${ }^{b}$ Department of Public Health and Caring \\ Sciences, Family Medicine and Preventive Medicine, Uppsala University, Uppsala, Sweden; 'The Swedish School of Sports and Health \\ Sciences, Stockholm, Sweden
}

\begin{abstract}
Background: Despite well-known preventive effects for future cardiovascular disease (CVD) risk through lifestyle changes, scientific evaluations of lifestyle programmes in primary care are scarce. Moreover, structured lifestyle counselling is still not integrated in everyday clinical practice. We aimed to evaluate change in cardiovascular risk factors and Framingham 10-year risk score of developing CVD in men and women at high cardiovascular risk after participation in a structured lifestyle programme over 1 year. A single-group study was carried out with a 1-year follow-up including before and after measurements.

Methods: The lifestyle programme comprised five appointments to a district nurse over 1 year, focussing on lifestyle habits based on motivational interviewing. Fasting blood samples and anthropometric measurements were obtained at baseline and 1-year follow-up. The 10-year risk of CVD was calculated according to Framingham general CVD risk score.

Results: A total of 404 patients were included in the study. There was a positive change over 1 year in the total study population for all risk factors evaluated. This included improvements in weight, waist circumference, blood pressure, blood lipids, and fasting glucose. The 10-year risk of developing CVD decreased for the total population from $24.8 \%$ to $21.4 \%$ at 1 year, equivalent to a $14 \%$ decrease.

Conclusions: A structured lifestyle programme in primary care contributes to significant improvements of cardiovascular risk factors and the reduction of 10-year risk for CVD for both men and women at high cardiovascular risk.
\end{abstract}

ARTICLE HISTORY

Received 23 August 2019

Revised 31 January 2020

Accepted 3 February 2020

\section{KEYWORDS}

Cardiovascular prevention; cardiovascular risk factors; Framingham heart study; hypertension; lifestyle counselling; type 2 diabetes mellitus

\section{Introduction}

Although there has been substantial improvement in cardiovascular disease (CVD) outcomes, non-communicable diseases including ischaemic heart disease, type 2 diabetes mellitus (T2DM), stroke, and chronic obstructive pulmonary disease account for the majority of deaths and disabilityadjusted life-years (DALYs) world-wide (1). The leading underlying risk factors in 2017 for death or DALYs were: high systolic blood pressure, causing 10.4 million deaths in 1 year, followed by smoking, high fasting plasma glucose, high body-mass index (BMI), and high concentrations of low-density lipoprotein cholesterol (LDL).

Guidelines from the American Heart Association, European Heart Association as well as the Swedish National Board of Health and Welfare emphasise that the highest clinical priority for CVD prevention should be directed towards patients with prevalent CVD or those at high risk of developing CVD (2-4). Despite the guidelines and the knowledge of the preventive effect for future CVD through lifestyle changes such as improved diet, increased physical activity, and smoking cessation, many patients with high CVD risk do not comply with this and have uncontrolled blood pressure and high concentrations of lipids and blood glucose (3,5-7). A teambased, comprehensive, patient-centred approach that addresses all aspects of a patient's lifestyle habits has previously been proposed as an effective strategy for CVD prevention in clinical practice $(4,8,9)$. However, scientific evaluations of lifestyle programmes in primary care are scarce (10-12), and structured lifestyle counselling is still not integrated in everyday clinical practice.

To enhance the care of patients recently diagnosed with hypertension, T2DM, or impaired glucose tolerance (IGT) and address their cardiovascular risk profile, we started a structured lifestyle programme at a primary care unit in Västerås, Sweden. In a previous publication, we observed favourable changes of lifestyle habits including physical activity, diet, smoking, and stress over 1 year (13). The results support the utility of a multifactorial, structured approach in clinical practice to change unhealthy lifestyle habits.

The aim of the present study was to evaluate changes in cardiovascular risk factors and 10-year risk for CVD according 
to the Framingham general CVD risk score in men and women at high cardiovascular risk after participation in a structured lifestyle programme in primary care for 1 year. We hypothesised that a structured lifestyle programme would reduce cardiovascular risk factors and 10-year risk of CVD.

\section{Methods}

\section{Study design and population}

We conducted a single-group study with a 1-year follow-up including before and after measurements. All data were collected consecutively and registered in a database. Patients registered at Citypraktiken, a primary care unit in Västerås, Sweden, were enrolled during a 5-year period between October 2009 and September 2014. Inclusion criteria were men and women aged 30-74 years, newly diagnosed with either hypertension (blood pressure $>140 / 90 \mathrm{mmHg}$ ), T2DM (fasting plasma glucose concentration $>7 \mathrm{mmol} / \mathrm{L}$ ), or IGT (2$\mathrm{h}$ glucose concentration of $7.8-11.0 \mathrm{mmol} / \mathrm{L}$ on the $75-\mathrm{g}$ oral glucose tolerance test). Antihypertensive or cholesterol-lowering medication was prescribed, when needed, according to hypertension and diabetes guidelines (14-16). Exclusion criteria were diagnosed dementia or severe psychiatric disease. A total of 448 patients were referred by their physician to join the lifestyle programme. Eleven patients did not meet the inclusion criteria, three patients died, and 30 patients did not provide consent. Thus, 404 patients were included in the analyses. Sixty-three patients (16\%) were lost to follow-up.

The study was conducted according to the ethical guidelines of the Declaration of Helsinki and the Good Clinical Practice guidelines. The Swedish Ethical Review Authority approved the study (reference number: 2014/497). All patients provided written informed consent. The study was registered at www.clinical-trials.gov (ClinicalTrial.gov ID: DNR 2014/497).

\section{The structured lifestyle programme}

The structured lifestyle programme has been described in detail previously (13). In short, the structured lifestyle programme comprised five appointments to a district nurse with post-graduate credits in diabetes care and the metabolic syndrome, at baseline and after 3, 6, 9, and 12 months. Every appointment focussed primarily on lifestyle habits. Fasting blood samples were obtained one week before the baseline and at the 1-year follow-up. Anthropometric variables were measured at baseline and at the 1-year follow-up. Blood pressure and waist circumference were measured at every appointment.

\section{Clinical examinations}

At baseline and 1-year follow-up, all patients were weighed to the nearest $0.1 \mathrm{~kg}$ using an electronic scale. Height was measured to the nearest $0.5 \mathrm{~cm}$. Body mass index was calculated from the measured weight and height as $\mathrm{kg} / \mathrm{m}^{2}$. Waist circumference was measured with the participant in a standing position, midway between the lower rib margin and the iliac crest to the nearest $0.5 \mathrm{~cm}$. Blood pressure was measured using a standard auscultatory method with the participant in a seated position after a 10-min rest.

\section{Laboratory measurements}

A blood sample was drawn from the antecubital vein after overnight fasting. Concentrations of total cholesterol (mmol/ L), low-density lipoprotein (LDL) ( $\mathrm{mmol} / \mathrm{L})$, high-density lipoprotein $(\mathrm{HDL})(\mathrm{mmol} / \mathrm{L})$, and triglycerides $(\mathrm{mmol} / \mathrm{L})$ were estimated by standard methods at Aleris MediLab (Stockholm, Sweden, ISO/IEC 151 89, certified). Fasting plasma glucose concentrations were analysed at the primary care unit from venous blood sample, using the HemoCue ${ }^{\circledR}$ Glucose 201 RT system.

\section{The Framingham 10-year risk of CVD}

Framingham general CVD risk score (2008) calculates the risk of developing cardiovascular events in 10 years (17). The prediction variables used are age, sex, total cholesterol, HDL cholesterol, systolic blood pressure, blood pressure treatment or not, diabetes mellitus or not, and current smoking. Endpoints assessed in the Framingham general CVD risk score are a composite of coronary heart disease (coronary death, myocardial infarction, coronary insufficiency, and angina), cerebrovascular events (including ischaemic stroke, haemorrhagic stroke, transient ischaemic attack), peripheral artery disease (intermittent claudication), and heart failure. A calculated 10-year risk of $<5 \%$ is considered as low, $\geq 5$ to $<7.5 \%$ as borderline, $\geq 7.5 \%$ to $<20 \%$ as intermediate, and $\geq 20 \%$ as high risk (18).

\section{The metabolic syndrome}

The metabolic syndrome was classified according to the National Cholesterol Education Programme (NCEP) Adult Treatment Panel III (ATPIII) (19) as $\geq 3$ of the following risk determinants; waist circumference $>102 \mathrm{~cm}$ (men) and $>88 \mathrm{~cm}$ (women); systolic blood pressure $\geq 130 / 85 \mathrm{mmHg}$; triglycerides $\geq 1.7 \mathrm{mmol} / \mathrm{L} ; \mathrm{HDL}<1.0$ (men) and $<1.3$ (women) $\mathrm{mmol} / \mathrm{L}$; or fasting plasma glucose $>6.1 \mathrm{mmol} / \mathrm{L}$. Patients with anti-hypertensive or statin medication have been included in the high blood pressure or triglyceride groups.

\section{The Care Need Index}

The Care Need Index is used to evaluate a population's need for primary care, with a high index indicating an increased need for health care (20). The Care Need Index measures socio-economic factors and comprises seven variables: age $>65$ years; born in Eastern Europe, Asia, Africa, or South America; unemployed; single parent with child younger than 17 years; children under 5 years; poor education level; and highly mobile people. 


\section{Statistics}

Results have been presented in the total population, by sex or by diagnosis (hypertension or T2DM + IGT). An intentionto-treat approach was used, and last observation was carried forward for missing data for all variables. Data were checked for normality by visual inspection of histograms for each variable. Parametric statistical methods should be used for all cardiovascular risk factors and Framingham risk score. A twotailed paired $t$ test was used to study within-group changes over 1 year, and an unpaired $t$ test to study subgroup differences. The rejection criteria for each of the individual nullhypotheses were adjusted according to the Bonferroni-Holm method to counteract the problem of multiple comparisons (i.e., type I error). Alpha-levels after correction with Bonferroni-Holm ranged from $p<0.005$ for the highest ranked result to $p<0.05$ for the lowest ranked result of the paired $t$ test. Chi-square test was used to study differences at baseline for medication and metabolic syndrome prevalence between study population and patients lost to follow-up. McNemar's test was used to detect changes over 1 year of proportions of patients at increased risk for each CVD risk factor and 10-year risk of CVD. Analyses were performed using Statistical Package for Social Science (IBM SPSS
Statistics for Windows, version 24.0; IBM Corp. Armonk, NY, USA).

\section{Results}

\section{Baseline characteristics}

The population comprised 404 participants (52\% women), median age 59 years (range 43-74 years), recently diagnosed with either hypertension (73\%), T2DM (25\%), or IGT $(2 \%)$ (Table 1). The metabolic syndrome was present in $50 \%$ of the study population, and $6 \%$ had a previous history of CVD. Subjects lost to follow-up during the study were slightly younger, more often daily smokers, and were more often treated with statins than patients in the study population $(p<0.05)$. The Care Need Index was 0.86 for the present primary care unit in 2014 as compared with $1.08( \pm 0.20)$ in the county of Västmanland.

\section{Cardiovascular risk factors}

There was a positive change over 1 year in the total study population for all risk factors evaluated (Table 2). Changes in systolic and diastolic blood pressure (BP) were observed in

Table 1. Baseline characteristics of the study population and patients lost to follow-up.

\begin{tabular}{|c|c|c|c|c|}
\hline & $\begin{array}{c}\text { Total sample } \\
(n=404)\end{array}$ & $\begin{array}{c}\text { Men } \\
(n=195)\end{array}$ & $\begin{array}{l}\text { Women } \\
(n=209)\end{array}$ & $\begin{array}{l}\text { Lost to follow-up } \\
\qquad(n=63)\end{array}$ \\
\hline Age, years & $59( \pm 8.8)$ & $59( \pm 9.0)$ & $60( \pm 8.6)$ & $55( \pm 10.1)^{a}$ \\
\hline Hypertension & $73 \%(294)$ & $65 \%(127)$ & $78 \%(162)$ & $79 \%(50)$ \\
\hline Daily smoking & $10 \%(41)$ & $9 \%(18)$ & $11 \%(23)$ & $19 \%(12)^{a}$ \\
\hline Anti-hypertensive medication & $56 \%(226)$ & $59 \%(114)$ & $54 \%(112)$ & $57 \%(36)$ \\
\hline Previous cardiovascular disease & $6 \%(25)$ & $8 \%(15)$ & $5 \%(10)$ & $6 \%(4)$ \\
\hline Metabolic syndrome & $50 \%(201)$ & $59 \%(114)$ & $42 \%(87)$ & $46 \%(29)$ \\
\hline
\end{tabular}

Data are presented as mean (SD) for age and \% (number of patients) for all other variables.

${ }^{\text {a }}$ Significantly different from study population $(p<0.05)$.

${ }^{b}$ Only individuals with type 2 diabetes mellitus or impaired glucose tolerance $(n=109)$ were included for this variable.

Table 2. Cardiovascular risk factors at baseline, 1 year, and change over 1 year, total sample.

\begin{tabular}{|c|c|c|c|c|}
\hline$n=404$ & $\begin{array}{l}\text { Baseline } \\
\text { mean (SD) }\end{array}$ & $\begin{array}{c}1 \text { year } \\
\text { mean (SD) }\end{array}$ & $\begin{array}{c}\text { Change over } 1 \text { year } \\
\text { mean }(95 \% \mathrm{Cl})\end{array}$ & $p$ Value \\
\hline Weight (kg) & $86.7(18.7)$ & $84.5(18.6)$ & $-2.1(-1.6 \text { to }-2.6)^{c}$ & $<0.001^{a}$ \\
\hline BMI $\left(\mathrm{kg} / \mathrm{m}^{2}\right)$ & $29.3(5.5)$ & $28.6(5.5)$ & $-0.7(-0.5 \text { to }-0.9)^{c}$ & $<0.001^{\mathrm{a}}$ \\
\hline Waist circumference $(\mathrm{cm})$ & $101.2(13.7)$ & $98.2(13.5)$ & $-2.9(-2.5$ to -3.4$)$ & $<0.001^{\mathrm{a}}$ \\
\hline Systolic BP (mmHg) & $149.2(17.1)$ & $140.5(15.4)$ & $-8.7(-7.1 \text { to }-10.4)^{c}$ & $<0.001^{\mathrm{a}}$ \\
\hline Anti-hypertensive medication, $n=268$ & $150.3(18.1)$ & $139.3(15.3)$ & $-11.0(-9.0 \text { to }-13.1)^{c}$ & $<0.001^{\mathrm{a}}$ \\
\hline No medication, $n=136$ & $147.0(14.8)$ & $142.8(15.5)$ & $-4.2(-1.7$ to -6.8$)$ & $0.001^{\mathrm{a}}$ \\
\hline Diastolic BP (mmHg) & $88.0(10.5)$ & $84.1(8.8)$ & $-4.0(-3.0 \text { to }-4.9)^{c}$ & $<0.001^{\mathrm{a}}$ \\
\hline Anti-hypertensive medication, $n=268$ & $88.4(11.1)$ & $83.3(8.6)$ & $-5.1(-3.9$ to -6.3$)$ & $<0.001^{\mathrm{a}}$ \\
\hline No medication, $n=136$ & $87.4(9.3)$ & $85.5(9.0)$ & $-1.8(-0.3 \text { to }-3.3)^{c}$ & $0.017^{\mathrm{a}}$ \\
\hline Total cholesterol (mmol/L) & $5.94(1.18)$ & $5.72(1.10)$ & $-0.22(-0.13$ to -0.31$)$ & $<0.001^{\mathrm{a}}$ \\
\hline With statin medication, $n=65$ & $6.19(1.63)$ & $5.22(1.36)$ & $-0.97(-0.58$ to -1.37$)$ & $<0.001^{\mathrm{a}}$ \\
\hline No statin medication, $n=339$ & $5.89(1.06)$ & $5.81(1.02)$ & $-0.08(0.00$ to -0.15$)$ & 0.051 \\
\hline $\mathrm{LDL}(\mathrm{mmol} / \mathrm{L})$ & $3.86(0.99)$ & $3.72(0.99)$ & $-0.14(-0.06$ to -0.21$)$ & $<0.001^{\mathrm{a}}$ \\
\hline With statin medication, $n=65$ & $3.89(1.32)$ & $3.21(1.19)$ & $-0.68(-0.38$ to -0.98$)$ & $<0.001^{\mathrm{a}}$ \\
\hline No statin medication, $n=339$ & $3.86(0.92)$ & $3.82(0.91)$ & $-0.04(-0.03$ to 0.10$)$ & 0.249 \\
\hline $\mathrm{HDL}(\mathrm{mmol} / \mathrm{L})$ & $1.37(0.37)$ & $1.40(0.38)$ & $0.04(0.02 \text { to } 0.05)^{c}$ & $<0.001^{\mathrm{a}}$ \\
\hline Triglycerides (mmol/L) & $1.66(1.06)$ & $1.51(0.82)$ & $-0.15(-0.08 \text { to }-0.23)^{b, c}$ & $0.001^{\mathrm{a}}$ \\
\hline Fasting plasma glucose (mmol), $n=109$ & $8.51(2.99)$ & $7.15(1.70)$ & $-1.36(-0.85$ to -1.88$)$ & $<0.001^{\mathrm{a}}$ \\
\hline Glucose-lowering medication, $n=42$ & $10.17(3.75)$ & $7.85(1.92)$ & $-2.32(-1.19$ to -3.46$)$ & $<0.001^{\mathrm{a}}$ \\
\hline No medication, $n=67$ & $7.48(1.75)$ & $6.72(1.39)$ & $-0.76(-0.35$ to -1.17$)$ & $<0.001^{\mathrm{a}}$ \\
\hline
\end{tabular}

Values are presented as mean (SD) for baseline and 1-year measurements, and as mean (95\% $\mathrm{Cl}$ ) for change over 1 year.

${ }^{\mathrm{a}}$ Significantly different after Bonferroni-Holm correction.

${ }^{\mathrm{b}}$ Significantly different between sex.

'Significantly different between diagnosis $(p<0.05)$. 
patients with or without anti-hypertensive medication, with the greatest decrease in patients with medication. Total cholesterol and LDL decreased mainly in patients treated with statin medication. Fasting plasma glucose decreased regardless of glucose-lowering medication for patients with T2DM or IGT. Longitudinal data for blood pressure and waist circumference (five measurements over 1 year) exposed a continuous decrease over 1 year (Supplementary Figures 1 and 2, available online).

Sex-specific subgroup analyses exposed positive changes for all risk factors, similar to the analysis of the total study group (Supplementary Table 1, available online).

Diagnosis-specific subgroup analyses exposed that all risk factors decreased for the two diagnosis subgroups, except for diastolic blood pressure and LDL in patients with T2DM or IGT. Reduction of systolic blood pressure was more prominent for patients with hypertension than with T2DM or IGT. Patients with T2DM or IGT had a larger reduction of weight and body mass index compared to patients with hypertension. Increases in HDL concentrations were more prominent for patients with T2DM or IGT (Supplementary Table 2, available on line).

Moreover, all analyses of cardiovascular risk factors have additionally been performed excluding participants lost to follow-up. The results are similar to the results from the study population where intention-to-treat was applied (Supplementary Tables 3-5, available online, for analyses of patients with data available at baseline and at 1 year).

\section{The Framingham 10-year risk of CVD}

The 10-year risk of developing CVD decreased for the total population over 1 year, equivalent to a relative decrease of $14 \%$ compared with baseline values (Table 3; Figure 1). Men had an almost doubled 10-year risk for CVD compared with women at both baseline and 1-year follow-up. Patients with T2DM or IGT had a higher 10-year risk than patients with hypertension. There was a decreased 10-year risk of developing CVD in both men and women, and for both diagnosis subgroups (Table 3; Supplementary Figure 3, available online).

\section{Change in proportions of patients at increased risk for different risk factors}

The proportions of patients at increased risk over 1 year decreased regarding blood pressure, triglycerides, body mass index, waist circumference, and Framingham risk score (Figure 2).

\section{Discussion}

We observed significant improvements for all risk factors and in 10-year CVD risk after participation in a 1-year structured lifestyle programme in primary care, in both men and women with high cardiovascular risk and irrespective of whether they were diagnosed with hypertension, T2DM, or IGT. This included improvements in weight, waist
Table 3. Estimated 10-year risk of developing CVD according to framingham risk score at baseline, 1 year, and change over 1 year.

\begin{tabular}{lcccc}
\hline & $\begin{array}{c}\text { Baseline, } \\
\text { \% Mean } \\
(\text { SD })\end{array}$ & $\begin{array}{c}1 \text { year, } \\
\text { \% Mean } \\
(\text { SD })\end{array}$ & $\begin{array}{c}\text { Change } \\
\text { over } 1 \text { year, } \\
\% \text { Mean } \\
(95 \% \mathrm{Cl})\end{array}$ & $p$ Value \\
\hline Total $(n=402)$ & $24.8(15.6)$ & $21.4(13.5)$ & $-3.4(-2.7$ to -4.1$)$ & $<0.001^{\text {a }}$ \\
Men $(n=193)$ & $31.8(16.8)$ & $27.9(14.2)$ & $-3.9(-2.7$ to -5.0$)$ & $<0.001^{\text {a }}$ \\
$\begin{array}{l}\text { Women }(n=209) \\
\text { Hypertension } \\
\quad(n=293)\end{array}$ & $21.3(11.2)$ & $15.4(9.6)$ & $-3.0(-2.1$ to -3.8$)$ & $<0.001^{\text {a }}$ \\
$\begin{array}{c}\text { T2DM + IGT } \\
(n=109)\end{array}$ & $34.8(18.7)$ & $18.3(11.2)$ & $-2.8(-2.1$ to -3.5$)$ & $<0.001^{\text {a }}$ \\
\hline
\end{tabular}

Values are presented as mean (SD) for baseline and 1-year measurements, and as mean $(95 \% \mathrm{Cl})$ for change over 1 year.

${ }^{a}$ Significantly different after Bonferroni-Holm correction.

${ }^{\mathrm{b}}$ Significantly different between diagnosis $(p<0.05)$.

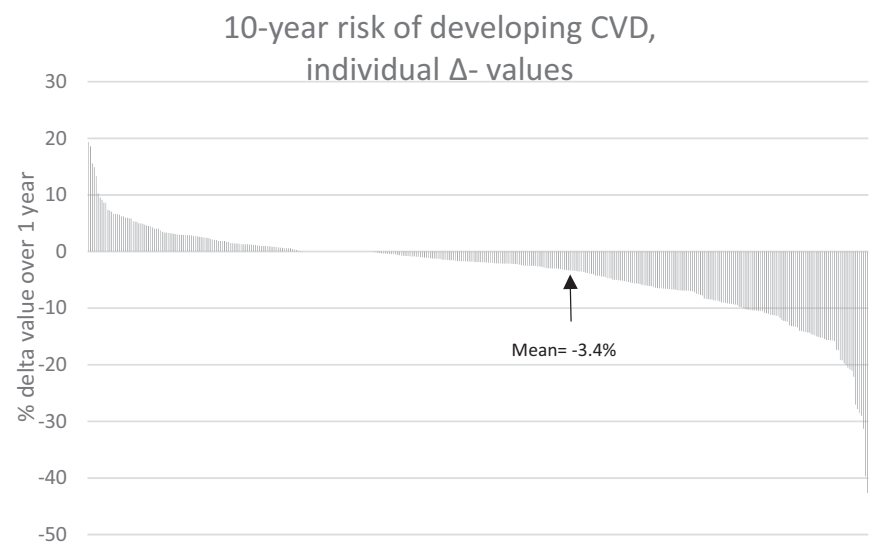

Figure 1. Ten-year risk of developing CVD, individual $\Delta$-values, total study population $(n=404)$.

circumference, blood pressure, blood lipids, and fasting glucose. The 10-year CVD risk decreased from $24.8 \%$ to $21.4 \%$ in the total study population equivalent to an absolute mean decrease of $3.4 \%$ in the total study population $3.0 \%$ in women and $3.9 \%$ in men). It is worthy of note that the total study population except for women were all at high risk for CVD, exceeding 20\% risk for future CVD. After participation in the lifestyle programme, patients with hypertension instead had an intermediate 10-year risk of CVD. Patients with T2DM + IGT had the highest 10-year risk at both the baseline and 1-year follow up, indicating that this subgroup is in crucial need of both drug therapy and lifestyle counselling.

The reduction in systolic blood pressure in our study is in line with previous studies. For example, a Swedish randomised study of 151 middle-aged men and women assigned to either lifestyle intervention or control group presented a $4.9 \mathrm{mmHg}$ reduction of systolic blood pressure and a $2.2 \mathrm{~cm}$ reduction of waist circumference in the intervention group compared with the control group (11). Similar results were reported in a review article by Dickinson et al. in which a mean reduction in systolic blood pressure of $5.0 \mathrm{mmHg}$ after participation in different lifestyle programmes was found (21). The US Preventive Services Task force presented comparable results in their updated version of a systematic 


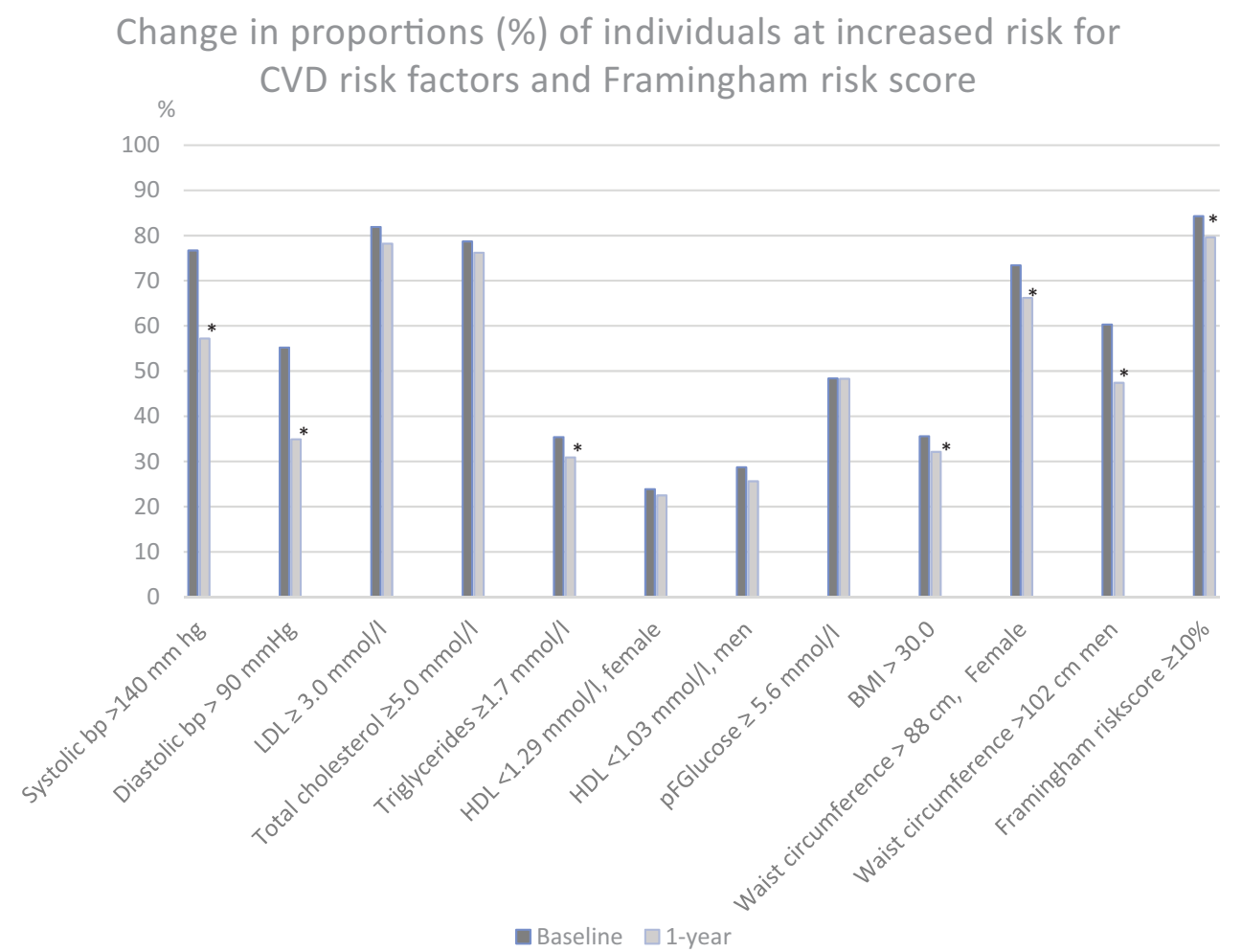

Figure 2. Change in proportions of patients at increased risk for CVD risk factors and Framingham risk score, total sample $(n=404)$. ${ }^{*}$ Significantly different from baseline after Bonferroni-Holm correction.

review regarding 'Behavioral counselling to promote a healthful diet and physical activity for cardiovascular disease prevention' (22). They concluded that even a small reduction in blood pressure $(2 \mathrm{mmHg}$ systolic blood pressure and $1 \mathrm{mmHg}$ diastolic blood pressure) may result in long-term decrease in CVD morbidity and mortality. Furthermore, epidemiological data suggest that this small reduction is associated with a decreased risk for coronary artery disease by $6 \%$ and for cerebrovascular event by $16 \%$ (23). In contrast, according to a report from the Swedish council on Health and Technology Assessment, $20 \%-30 \%$ of those who have been prescribed blood pressure-lowering drugs do not reach the treatment goal of blood pressure $<140 / 90 \mathrm{mmHg}$ (24). The results of our study indicate that the number of patients that reach the blood pressure goal of $<140 / 90$ can be increased with a structured lifestyle programme and counselling, regardless of anti-hypertensive medication or not.

Patients receiving statin medication showed reduced serum concentrations of cholesterol and LDL, which is in line with previous observations suggesting fairly low effects of reduced or modified dietary fat on serum (25). According to the AHA/ACC guidelines to prevent CVD, all patients at intermediate and high risk of CVD should be given statin treatment aiming to reduce serum LDL by $30 \%-40 \%$ and $50 \%$ respectively (2). Similar recommendations are also stated in the National Guidelines for Diabetes Care from the Swedish National Board of Health and Welfare (26). It is also stressed that enhancement of lifestyle habits should always accompany medical treatment, for all patients at high cardiovascular risk (2), which is in agreement with the intention of the structured lifestyle programme in our study.
An increase of HDL was detected in the total study population as well as in sex and diagnosis subgroups. This was most prominent for patients with T2DM. It is well known that the serum concentration of HDL depends on to which extent a person is physically active, and that increased physical activity increases serum HDL (27). In our previous study on how lifestyle habits changed after participating in the structured lifestyle programme we observed an increase in physical activity, which could be an explanation of the present increase of HDL (13).

The relative decline by $14 \%$ of the 10-year risk of CVD in the total study population is similar to another Swedish study of 100 patients at high cardiovascular risk who participated in a 1-year lifestyle programme presenting a 15\% decrease over 1 year (28). On the other hand, there are diverse results of the effects on 10-year risk of CVD after participating in lifestyle counselling. In a Canadian randomised controlled treatment study consisting of 315 patients, the authors reported a $24 \%$ decreased 10 -year risk after participating in a telehealth counselling for patients at high cardiovascular risk (29). In contrast, a study from the Finnish diabetes prevention programme with 2250 patients presented a $2.5 \%$ decreased 10-year risk after lifestyle counselling offered in primary health care (30). The intensity of the lifestyle programme and groups targeted are partly different from the population in our study, which may explain the various results, although we find it promising that the 10year risk of CVD can be reduced. Despite the modest reduction in the Finnish study, the authors calculated that 25 men or 59 women would need to change their lifestyle to prevent one CVD in 1 year (30). 
This study was carried out in an ordinary primary care setting, performed in everyday practice, using only the limited resources available at the clinic. These 'real world data' strengthen the external validity even though the lack of randomisation limits the inference of the results. As the lack of control group limits the analyses of casual relationship between participation in the programme and change in cardiovascular risk, regression towards the mean must be considered. Although the results of repeated measurements of blood pressure and waist circumference may indicate a possible relationship to the reduced CVD risk seen in our material (as the regression towards the mean effect gets smaller with repeated measurements), this limitation has to be taken into account when interpreting our results. This is an intention-to-treat study, where $16 \%$ of the patients were lost to follow-up. As this may influence the interpretation of the results, we completed an analysis of the data excluding patients lost to follow-up. The results regarding change in cardiovascular risk factors and CVD risk over 1 year for the study population excluding participants lost to follow-up were well in line with the results where intention-to-treat analysis was applied. We therefore find that the results of the intention-to-treat analysis are valid, despite the fact that there were participants lost to follow-up.

As lifestyle counselling is a heterogeneous phenomenon, an evaluation of a real-life setting is of importance. The high socio-economic index for the family care unit in this study might impair the generalisation to other family care units in less prosperous communities. We find that the study design and the rather large number of patients included in our study give an indication of what effect a structured lifestyle programme carried out in primary care might have on cardiovascular risk factors and 10-year risk of CVD. As all patients with a recent diagnosis with hypertension, T2DM, or IGT were invited to participate in the structured programme, the population studied is a typical representation of patients receiving care at a primary care unit. It also indicates that the intervention is feasible to carry out in a primary care setting and might be implemented at other primary care units.

We conclude that a structured lifestyle programme in primary care contributes to significant improvements of cardiovascular risk factors and the reduction of 10-year risk for CVD for both men and women at high cardiovascular risk. Since this is a single-group study there is a need for future randomised controlled trials to confirm our findings.

\section{Acknowledgements}

We thank all staff at Citypraktiken, in particular the nurses who performed all counselling sessions. We also thank Professor Mai-Lis Hellenius for the inspiration to start the structured lifestyle programme.

\section{Disclosure statement}

The authors report no conflict of interest.

\section{Funding}

Funding was received from Praktikertjänst AB, 10355 Stockholm.

\section{Notes on contributors}

Lena Lönnberg physiotherapist, doctoral student at Centre for Clinical Research, Västerås, Sweden; main research focus on lifestyle counselling for patients at high cardiovascular risk.

Elin Ekblom-Bak, associate professor at The Swedish School of Sport and Health sciences in Stockholm, Sweden; main research focus on the association between sedentary behaviour, physical activity, and cardiorespiratory fitness on health and disease risk in the adult population.

Mattias Damberg, associate professor at Uppsala University, Uppsala, Sweden; main research focus on the cardiovascular prevention.

\section{ORCID}

Lena Lönnberg (D) http://orcid.org/0000-0003-4706-6915

Elin Ekblom-Bak (iD) http://orcid.org/0000-0002-3901-7833

Mattias Damberg (iD http://orcid.org/0000-0001-7654-7553

\section{References}

1. Stanaway JD, Afshin A, Gakidou E, Lim SS, Abate D, Abate $\mathrm{KH}$, et al. Global, regional, and national comparative risk assessment of 84 behavioural, environmental and occupational, and metabolic risks or clusters of risks for 195 countries and territories, 1990-2017: a systematic analysis for the Global Burden of Disease Study 2017. Lancet. 2018;392:1923-94. doi:10.1016/S01406736(18)32225-6

2. Arnett DK, Blumenthal RS, Albert MA, Buroker AB, Goldberger ZD, Hahn EJ, et al. ACC/AHA guideline on the primary prevention of cardiovascular disease. Circulation. 2019;140:596-646.

3. Kotseva $K$, De Bacquer D, De Backer G, Rydén L, Jennings $C$, Gyberg V, et al. Lifestyle and risk factor management in people at high risk of cardiovascular disease. A report from the European Society of Cardiology European Action on Secondary and Primary Prevention by Intervention to Reduce Events (EUROASPIRE) IV cross-sectional survey in 14 European regions. Eur J Prev Cardiol. 2016;23:2007-18. doi:10.1177/2047487316667784

4. The National Board of Health and Welfare. National guidelines for prevention and treatment of unhealthy lifestyle. Stockholm: The National Board of Health and Welfare; 2018.

5. The Public Health Agency of Sweden. Nationella folkhälsoenkätenHälsapålikavillkor. Stockholm. The Public Health Agency of Sweden; 2017 [Cited 2019 April 29]. Available from: https://www. folkhalsomyndigheten.se/folkhalsorapporteringstatistik/statistikdatabaser-och-visualisering/nationella-folkhalsoenkaten/levnadsvanor/fysisk-aktivitet/2016.

6. Folsom AR, Yatsuya $H$, Nettleton JA, Lutsey PL, Cushman $M$, Rosamond WD. Community prevalence of ideal cardiovascular health, by the American Heart Association definition, and relationship with cardiovascular disease incidence. J Am Coll Cardiol. 2011;57:1690-6. doi:10.1016/j.jacc.2010.11.041

7. Turco JV, Inal-Veith A, Fuster V. Cardiovascular health promotion: an issue that can no longer wait. J Am Coll Cardiol. 2018;72: 908-13. doi:10.1016/j.jacc.2018.07.007

8. Kotseva K, Wood D, De Bacquer D, De Backer G, Rydén L, Jennings $C$, et al. EUROASPIRE IV: a European Society of Cardiology survey on the lifestyle, risk factor and therapeutic management of coronary patients from 24 European countries. Eur J Prev Cardiolog. 2016;23:636-48. doi:10.1177/2047487315569401

9. Melvin $C L$, Jefferson MS, Rice LJ, Nemeth LS, Wessel AM, Nietert PJ, et al. A systematic review of lifestyle counseling for diverse patients in primary care. Prev Med. 2017;100:67-75. doi:10.1016/j. ypmed.2017.03.020

10. Lin JS, O'Connor EA, Evans CV, Senger CA, Rowland MG, Groom HC, U.S. Preventive Services Task Force evidence syntheses, formerly systematic evidence reviews. Behavioral counseling to promote a healthy lifestyle for cardiovascular disease prevention in 
persons with cardiovascular risk factors: an updated systematic evidence review for the US Preventive Services Task Force. Rockville (MD): Agency for Healthcare Research and Quality (US); 2014.

11. Eriksson MK, Franks PW, Eliasson M. A 3-year randomized trial of lifestyle intervention for cardiovascular risk reduction in the primary care setting: the Swedish Bjorknas study. PLoS One. 2009;4: e5195. doi:10.1371/journal.pone.0005195

12. Ketola E, Makela M, Klockars $M$. Individualised multifactorial lifestyle intervention trial for high-risk cardiovascular patients in primary care. Br J Gen Pract. 2001;51:291-4.

13. Lonnberg L, Ekblom-Bak E, Damberg M. Improved unhealthy lifestyle habits in patients with high cardiovascular risk: results from a structured lifestyle programme in primary care. Ups J Med Sci. 2019;124:94-104. doi:10.1080/03009734.2019.1602088

14. The National Board of Health and Welfare. Nationella riktlinjer för diabetesvård: stöd för styrning och ledning. Stockholm: The National Board of Health and Welfare; 2010.

15. Graham I, Atar D, Borch-Johnsen K, Boysen G, Burell G, Cifkova R, et al. European guidelines on cardiovascular disease prevention in clinical practice: executive summary: Fourth Joint Task Force of the European Society of Cardiology and other societies on cardiovascular disease prevention in clinical practice (constituted by representatives of nine societies and by invited experts). Eur Heart J. 2007;28:2375-414. doi:10.1093/eurheartj/ehm316

16. Swedish Agency for Health Technology Assessment and Assessment of Social Services. Måttligt förhöjt blodtryck uppdatering 2007. En systematisk litteraturöversikt. Stockholm; 2007.

17. D'Agostino RB, Sr, Vasan RS, Pencina MJ, Wolf PA, Cobain M, Massaro JM, et al. General cardiovascular risk profile for use in primary care: the Framingham Heart Study. Circulation 2008;117: 743-53. doi:10.1161/CIRCULATIONAHA.107.699579

18. Lloyd-Jones DM, Braun LT, Ndumele CE, Smith SC, Jr, Sperling LS, Virani SS, et al. Use of risk assessment tools to guide decisionmaking in the primary prevention of atherosclerotic cardiovascular disease. Circulation 2019;139:1162-77. doi:10.1161/ CIR.0000000000000638

19. National Cholesterol Education Program (NCEP). Expert Panel on Detection, Evaluation, and Treatment of High Blood Cholesterol in Adults (Adult Treatment Panel III). Third report of the National Cholesterol Education Programme (NCEP) expert panel on detection, evaluation, and treatment of high blood cholesterol in adults (adult treatment panel III) final report. Circulation 2002;106: 3143-421.

20. Malmstrom M, Sundquist J, Bajekal M, Johansson SE. Indices of need and social deprivation for primary health care. Scand J Soc Med. 1998;26:124-30. doi:10.1177/14034948980260021301
21. Dickinson HO, Mason JM, Nicolson DJ, Campbell F, Beyer FR, Cook $J V$, et al. Lifestyle interventions to reduce raised blood pressure: a systematic review of randomized controlled trials. J Hypertens. 2006;24:215-33. doi:10.1097/01.hjh.0000199800.72563.26

22. Grossman DC, Bibbins-Domingo K, Curry SJ, Barry MJ, Davidson $\mathrm{KW}$, Doubeni CA, et al. Behavioral counseling to promote a healthful diet and physical activity for cardiovascular disease prevention in adults without cardiovascular risk factors: US Preventive Services Task Force Recommendation Statement. JAMA 2017;318: 167-74. doi:10.1001/jama.2017.7171

23. Patnode CD, Evans CV, Senger CA, Redmond N, Lin JS. Behavioral counseling to promote a healthful diet and physical activity for cardiovascular disease prevention in adults without known cardiovascular disease risk factors: updated evidence report and systematic review for the US Preventive Services Task Force. JAMA 2017; 318:175-93. doi:10.1001/jama.2017.3303

24. Assessment SCoHT. Moderately elevated blood pressure: a systematic review summary and conclusions SBU Yellow Report No. 170/ 1U. Stockholm: Swedish Council on Health Technology Assesment (SBU); 2008.

25. Hooper L, Summerbell CD, Thompson R, Sills D, Roerts FG, Moore $\mathrm{HJ}$, et al. Reduced or modified dietary fat for preventing cardiovascular disease. Cochrane Database Syst Rev 2012;2012:CD002137.

26. The National Board of Health and Welfare. Nationella riktlinjer för diabetesvård: stöd för styrning och ledning. Stockholm: The National Board of Health and Welfare; 2015.

27. Zhang X, Devlin HM, Smith B, Imperatore G, Thomas W, Lobelo F, et al. Effect of lifestyle interventions on cardiovascular risk factors among adults without impaired glucose tolerance or diabetes: a systematic review and meta-analysis. PLoS One. 2017;12:e0176436. doi:10.1371/journal.pone. 0176436

28. Lidin M, Hellenius ML, Rydell-Karlsson M, Ekblom-Bak E. Long-term effects on cardiovascular risk of a structured multidisciplinary lifestyle program in clinical practice. BMC Cardiovasc Disord. 2018;8: 59. doi:10.1186/s12872-018-0792-6

29. Wister A, Loewen N, Kennedy-Symonds H, McGowan B, McCoy B, Singer J. One-year follow-up of a therapeutic lifestyle intervention targeting cardiovascular disease risk. Cmaj 2007;177:859-65. doi: 10.1503/cmaj.061059

30. Rautio N, Jokelainen J, Pölönen A, Oksa H, Peltonen M, Vanhala M, et al. Changes in lifestyle modestly reduce the estimated cardiovascular disease risk in one-year follow-up of the Finnish diabetes prevention programme (FIN-D2D). Eur J Cardiovasc Nurs. 2015;14: 145-52. doi:10.1177/1474515114521713 